\title{
Ensuring Movement Safety for Ground, Airborne and Sea Vehicles
}

\author{
Andrey Ananenkov, Anton Konovaltsev, Vladimir Nuzhdin, Vladimir Rastorguev, and Pavel Sokolov \\ Moscow Aviation Institute (National Research University), Moscow, Russia
}

https://doi.org/10.26636/jtit.2018.127018

\begin{abstract}
This article presents the features of an all-weather radio vision system (RVS) ensuring the safety of movement of ground, airborne and sea vehicles and automation of vehicle traffic control under limited or non-existent visibility conditions. New and promising RVS applications in the aviation and rail transport sectors are presented. The potential use of RVS based on an interferometric radar with aperture synthesis, capable of estimating the position of ice fields and the height of icebergs is considered as well.
\end{abstract}

Keywords-automotive radio vision system, full-scale tests, interferometric radar with aperture synthesis, traffic control automation, traffic safety.

\section{Introduction}

One of the urgent problems that needs to be faced in the development of modern vehicles is to ensure the safety and automation of controlling the traffic of both manned and autonomous (unmanned) vehicles under limited or no optical visibility conditions. This problem is equally relevant for road, air, water (sea and river) and rail transport. However, the specific nature of each of those modes of transport is linked with additional requirements that need to be met by the safety sensors.

In road transport, as evidenced by the results of research conducted by NTHSA (USA) [1], in addition to vehicle malfunction, $68 \%$ of road accidents are caused by inattention and inadequate awareness of the driver about the traffic situation. These are directly dependent on the visibility of the roadway, on the presence of markings and signs, on the degree of contamination of the cabin glazing, on the presence of fog, snow, rain, dust, smoke and other disturbing factors.

Attempts to solve the driver awareness problem using sensors that operate based on various physical principles (ultrasonic, optical and infrared) are known to have been made, but they failed to provide any major breakthroughs. The drawback of these solutions is obvious - of all negative factors that reduce optical visibility are linked to insufficient illumination. Thus, the main way to solve the actual problem of improving the safety of road transport, under limited or non-existent optical visibility conditions, is to use a radar sensor whose operation does not depend on the time of the day, on weather conditions (snow, rain, fog), or on the presence of smoke or dust [2]-[9]. The range of such a sensor can be a multiple of the expected length of the stopping distance.

The task of creating a compact radar that can be used in a car, enabling the driver to continue driving under insufficient visibility conditions (dense fog) has been successfully solved by a team of researchers from the Department of Radio Receivers of the Moscow Aviation Institute (MAI). In 2001, the first model of the automotive radio vision system (ARVS), relying on the $\mathrm{W}$ band $(\lambda=4 \mathrm{~mm}$ ) and approved for this class of radar by the EU and USA, was created. The use of a new holographic antenna, digital signal processors and an LCD indicator made it possible to reduce the weight and the dimensions of ARVS, so that it closely resembles infrared (IR) systems with respect to these parameters. The system can be easily installed on almost any car [10].

The market of rapidly developing automobile safety systems is not the only one where demand for small-size radar sensors exists. In order to enter the segment of safety systems used on small aircraft and/or in rail transport, it is necessary to significantly increase the operational range of ARVS. To ensure the safety of sea/river transport in icing conditions, it is also necessary to ensure that the radar sensor has the capability to estimate the height of ice formations.

As shown by the results of research focusing on ARVS [10]-[12], it is possible to increase the system's operational range to $1500 \mathrm{~m}$ and even to $3000 \mathrm{~m}$ without any major changes involving technology. The increase in operational range of ARVS will expand the scope of its application.

This version of RVS may be used:

- for ensuring the safety of take-off, landing and cruise phases of light and ultra-light aircraft, which do not currently have any radar equipment, in conditions of limited optical visibility;

- for improving the safety of railway traffic in under limited or non-existent optical visibility conditions, including at level crossings with roads;

- for solving ship navigation-related problems:

- berthing, including in automated mode,

- passage under bridges or through other "bottle necks", 
- passage through locks,

- high-precision navigation within ports premises,

- detection of semi-submerged objects,

- solution of the problem of the "blind" zone of regular navigational radars,

- detection of ice sheets and icebergs.

In this article, we consider the technical specifications of such a prospective sensor, and present a corresponding model - created at MAI (NIU) - a technology demonstrator, describing the research and field tests conducted. The successful creation of a sample radio vision system (RVS) having the form of a panoramic short-range radar (SRR) that meets the requirements applicable to road transport use, has led to the introduction of such RVSs in aviation, water and rail transport. Further development of radio vision systems used in water transport is linked to the creation of an interferometric radar with the synthesis of the antenna aperture (SAR). This will make it possible to estimate the height of ice surfaces, and detect dilution and icebergs, thus solving the problem of safe navigation of vessels in icing conditions. In this article, estimates of the potential characteristics of such a system, based on ARVS, are given.

\section{Aviation RVS for Flight Safety}

One of the actual problems faced while developing modern small aircraft is to ensure the safety of flight by preventing mid-air collisions, as well as by enabling to perform safe low-altitude flights, taxiing, take-offs and landings under limited optical visibility conditions. However, due to their low take-off weight, small aircraft are not equipped with radar. The increase in the working range of radio vision systems, with the acceptable weight and size characteristics maintained, has made it possible to propose to develop a radar sensor suitable for small aircraft.

The result of the project implemented jointly by JSC "Techaviakompleks" (Zhukovsky, Moscow region) and the

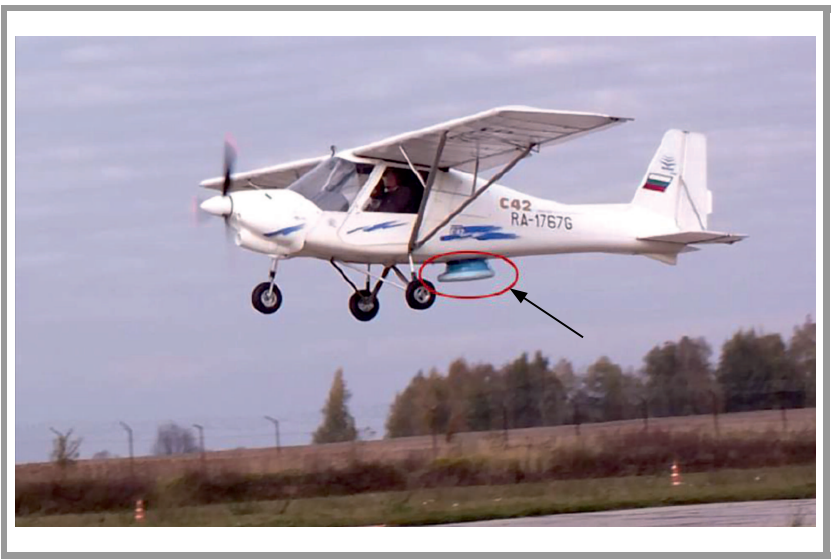

Fig. 1. IKARUS C42 plane with aviation-grade RVS, during testing. research team of MAI's Department of Radio Receiving Devices in 2016 was a model of an onboard safety system that is mounted on small aircraft and used to prevent mid-air collisions and to ensure safe performance of a lowaltitude flights, with the use of a small-size airborne radar complex (RVS) [13]-[14].

The RVS solution considered (Fig. 1) is of the navigational two-coordinate SRR variety, which determines a number of radar information processing and presentation-related features.

The key parameters of the experimental RVS model intended for the aviation industry are listed in Table 1.

Table 1

Key specifications of aviation-grade RVS

\begin{tabular}{|l|c|}
\hline Instrumental range & $20-2000 \mathrm{~m}$ \\
\hline Range resolution & $1.5 \mathrm{~m}$ \\
\hline Resolution azimuth & $\geq 1^{\circ}$ \\
\hline Operating frequency & $39 \mathrm{GHz}$ \\
\hline Radiation power & waveguide-slot \\
\hline Antenna & $\pm 60^{\circ}\left(360^{\circ}\right)$ \\
\hline Antenna-view in the azimuth plane & $\begin{array}{c}\text { from }+10^{\circ} \text { (up) } \\
\text { to }-40^{\circ}(\text { down })\end{array}$ \\
\hline Antenna-view in elevation plane & $256 \times 1024$ for shot \\
\hline $\begin{array}{l}\text { The number of information points } \\
\text { of the radar image }\end{array}$ & $\begin{array}{c}<100 \mathrm{~W}(12-24 \mathrm{~V} \\
\text { power supply) }\end{array}$ \\
\hline Energy consumption & $5 \div 10 \mathrm{~Hz}$ \\
\hline Information update speed & $<10 \mathrm{~kg}$ \\
\hline Radar weight &
\end{tabular}

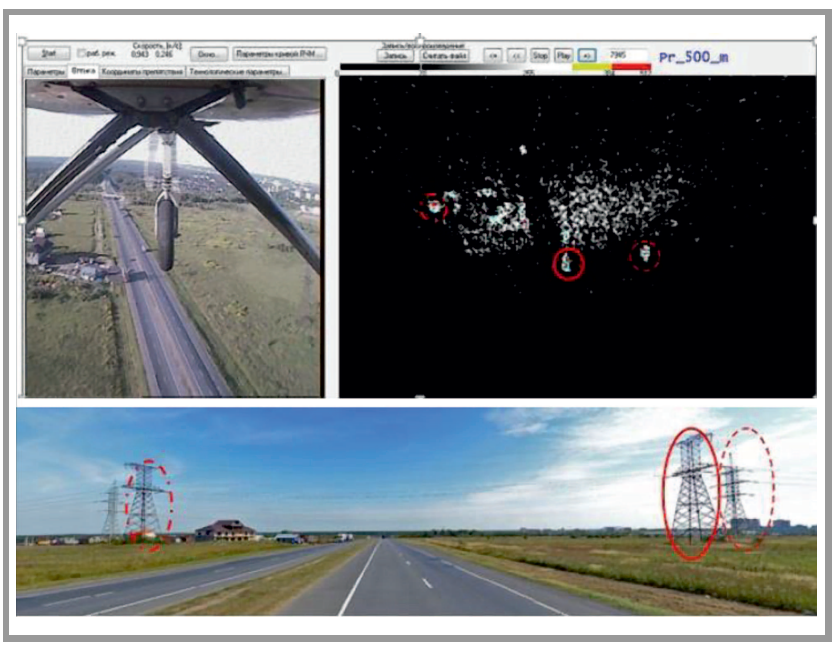

Fig. 2. Radar and video images of a location with power transmission lines (highlighted in red) and low-rise buildings. (For color pictures visit www.nit.eu/publications/journal-jtit)

The technical characteristics and functional capabilities of the modernized RVS have confirmed, during the test flights, that the following tasks can be performed undelimited 
(a)

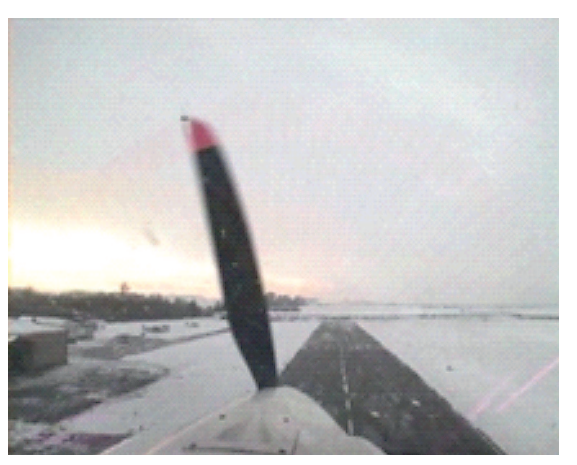

(b)

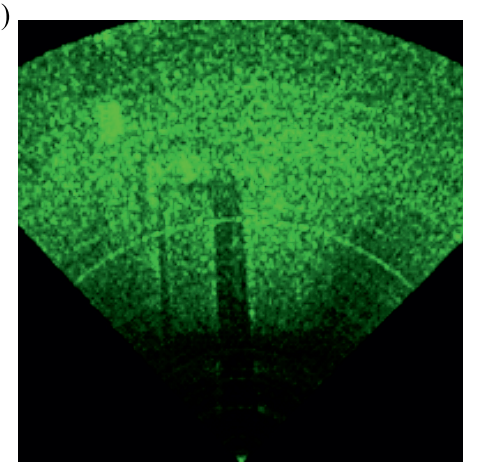

(c)

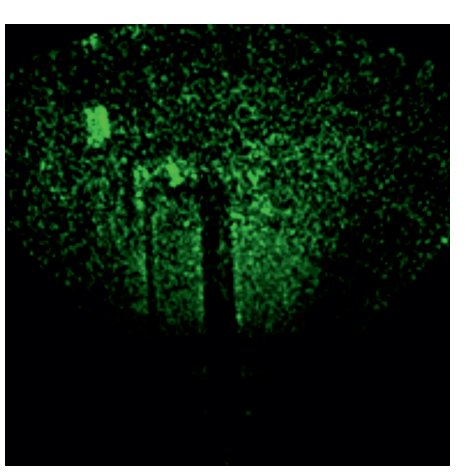

Fig. 3. Images of the runway (during take-off): (a) video, (b) radar, and (c) radar with noise filtration.

optical visibility conditions (twilight, fog, drizzle, snow, smoke, etc.):

- airspace control for the presence of airborne objects (helicopters, airplanes, gliders, etc.) and high-rise ground-based objects (relay towers, factory chimneys, tall buildings, etc.), creation of the outline of those objects (Fig. 2);

- formation of highly informative and detailed radar images of the Earth's surface (mapping), enabling the aircraft's navigation systems to rely on the radar map, to search for landing sites, to "see" the runway and taxiways (Fig. 3);

- detection of fixed obstacles and moving vehicles present on runways and taxiways, while landing and taxiing.

The aviation-grade RVS that has been developed provides radar data information that can be presented on the multifunctional display in real time [13]-[14].

\section{Application of RVS in Rail Transport}

One of the actual problems faced in modern rail transport is related to the improvement of railway traffic safety under limited or zero visibility conditions. With quick development of high-speed and automated railway transport systems observed today, the hazards posed by potentially dangerous situations increase substantially:

- unauthorized and uncontrolled presence on crossing of tracks by: people, animals, foreign objects,

- emergency situations related to the presence of wagons, locomotives, rail cars and road vehicles on the tracks, within the area of unguarded level crossings,

- consequences of flooding and blurring the roadway.

One of the most effective methods of improving the safety of rail transport is the use of a radar-optical complex (ROC) located on the locomotive and including a short-range RVS radar and an optical sensor. Both devices use a shared display and enable remote detection of obstacles on the tracks:

- under limited zero visibility conditions,

- regardless of weather conditions and of time of day,

- at railway stations - against a background of intense reflections from buildings, structures and the underlying surface,

- in a programmable controlled/monitoring zone,

- in the presence of numerous objects.

IDS-76 - a radar-based range and speed measurement device used in rail transport and developed by DOC (St. Petersburg, Russia) [15] is an example of a system relied upon to improve rail transport safety. The IDS-76 radar is installed permanently on the railway track, for example, at the end of the track (dead-end) and measures the movement parameters of the approaching rolling stock (speed, distance) and transfers this data to the PRISMA-K integrated traffic safety system. It is used at dead-end tracks of passenger stations and protects high-speed passenger suburban traffic and shunting works. The PRISMA-K system determines that a given approach is dangerous and transfers the data and relevant commands to the driver, demanding speed reduction or forcing the locomotive or the head car of the approaching train to perform an automatic stop (Fig. 4).

The IDS-76 radar is based on a $76 \mathrm{GHz}$ car radar and its detection range equals 1.5 to $500 \mathrm{~m}$, with the speed range of $1-100 \mathrm{~km} / \mathrm{h}$. Due to the short operating range of this type of radar, millimeter waves are only slightly attenuated by fine-dispersed interference in the form of smoke, dust and precipitation. In other words, the IDS-76 radar suffers from practically no weather - related distortions under the range of $500 \mathrm{~m}$.

The diameter of the antenna, including the radio-transparent cap, is $600 \mathrm{~mm}$. The radar has, with its wavelength considered, a very narrow beam width of $0.42^{\circ}$. This makes 

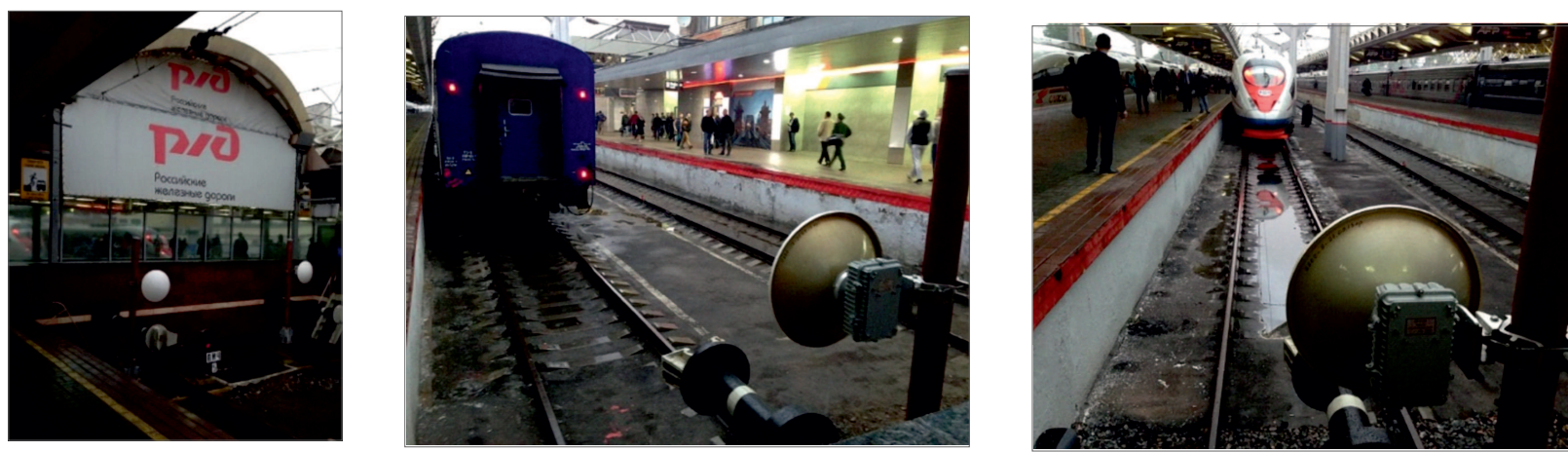

Fig. 4. IDS-76 radar.

it possible to control only those objects that are within the line of sight on a given track, without reacting trains arriving on adjacent tracks, or passengers and vehicles present on the platform.

Obviously, this radar station, positioned on a permanently fixed barrier, increases safety at braking and stopping stage of the journey, but not while traveling at speed. However, positive experience concerning the IDS-76 radar confirms the relevance of using radar in railway applications.

To ensure a higher level of safety along the route, the following systems should be incorporated into rail transport ROC solutions:

- panoramic front-end short-range radar,

- optoelectronic system with television and/or infrared channels,

- sound and visual warning system for the driver,

- channels transmitting information and communications to traffic supervisors,

- server for data processing, registration and storage,

- optionally - an emergency braking system.

Expert assessment of the Russian Railways (JSC "RZD”) specialists enabled RVS - MAI designers to formulate the following requirements related to the main ROC characteristics:

- size of the controlled security zone: person on the tracks detection range of $400 \mathrm{~m}$, vehicle on the tracks detection range of $800 \mathrm{~m}$, train detection range of $1000 \mathrm{~m}$,

- size of the scanned area, within the angle of $\pm 30^{\circ}$, and with the elevation angle of $\pm 5^{\circ}$,

- information update rate of $5-10 \mathrm{~Hz}$,

- number of objects to be detected - not less than 3 ,

- priority of objects according to the criterion of shortest distance,

- RVS is interfaced with optoelectronic facilities,
- output of information about the object and the presentation of a visual/audible signal when obstacles are detected on the railway tracks within the safety zone,

- RVS positioned on the roof of the locomotive, under a radio-transparent cover, with an indicator mounted in the driver's cab,

- environmental safety.

Obviously, the given technical requirements make it feasible to design a similar RVS based on automotive-grade RVS (ARVS), which is discussed in detail in [12].

At the end of 2016, tests demonstrating RVS developed by MAI (NRU) for railway transport application were carried out. Standard electric trains made by the Gorky branch of "RZD" were used. The tests were performed both in stationary mode (within the depot area), and with the electric train moving at a purpose-designed pattern.

In static tests, two variants of RVS placement were tested: on the coupling and on the roof of the electric train's locomotive. During dynamic tests in motion, the placement that was possible in such a scenario was used - on the roof of the electric train's locomotive. Figure 5 shows a photograph of the control module and the RVS display located in the driver's cab. The results of testing the railway version of RVS are shown in Figs. 6-11.

Figure 6 shows a synchronous optical (OI) and radar image (RI) of railway tracks, with a person who is near the tracks, at different distances. In this situation, the radar was placed on the roof of the locomotive. The mark indicating the person on the tracks (highlighted) in OI and RI images proves that the solution is stable at the range of $120 \mathrm{~m}$. Figures 7 and 8 show OI and RI images of railway tracks, with a person who is near the tracks (highlighted), as well as near the locomotive (highlighted).

It has been established, based on the tests results obtained with the electric train moving (Fig. 9), that the experimental RVS system:

- generates a highly detailed RI image in real-time,

- enables observation and detection of people walking along and standing on the tracks, 

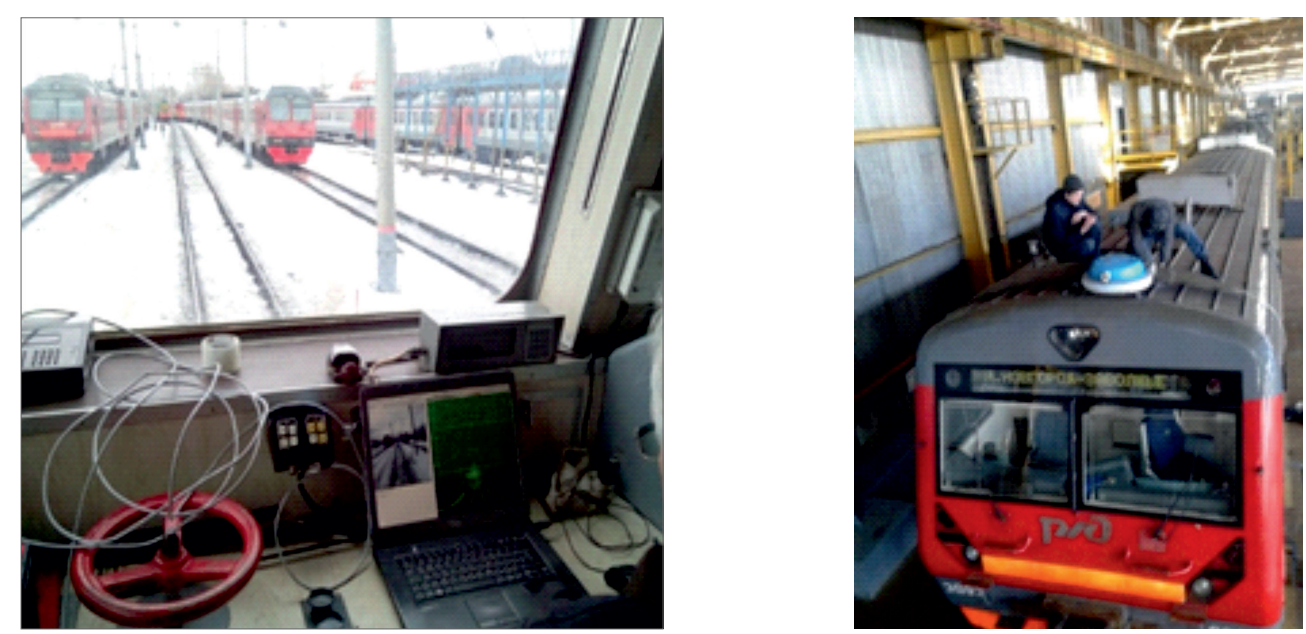

Fig. 5. Control and display modules in the driver's cab, with the radar placed on the roof.

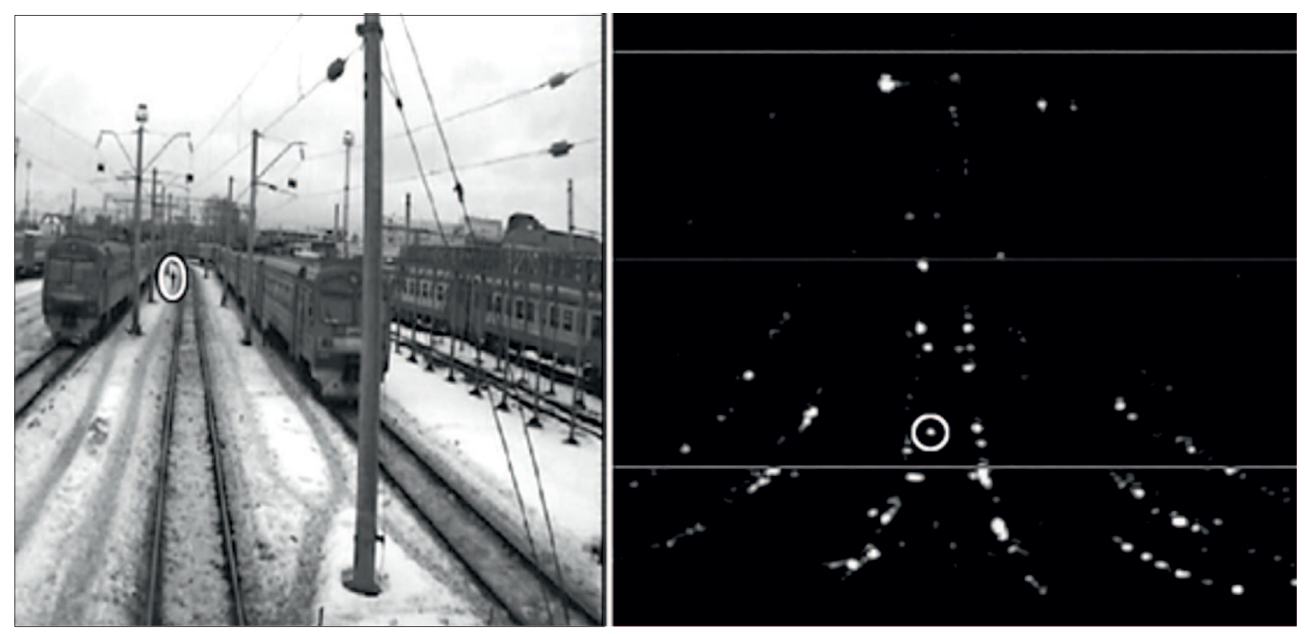

Fig. 6. OI and RI images (vertical coordinate - azimuth, horizontal - range) of railway tracks with a man 120 m away (highlighted).

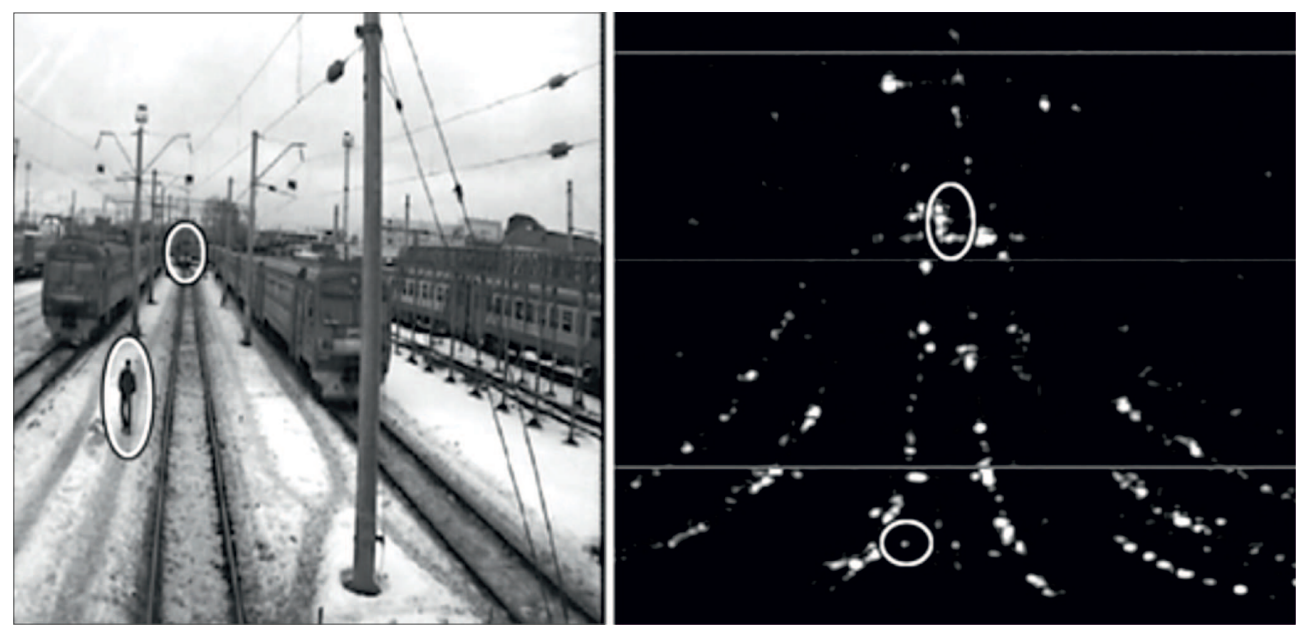

Fig. 7. OI and RI images of railway tracks with a man and a locomotive (highlighted). 


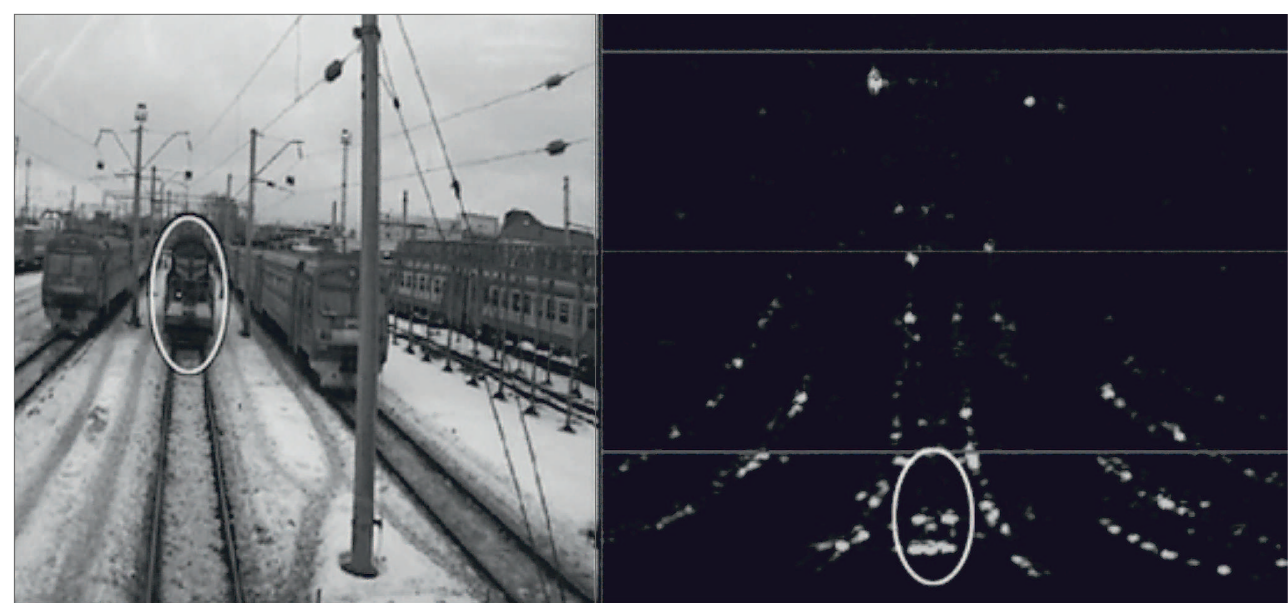

Fig. 8. OI and RI images of railway tracks with a Diesel locomotive (highlighted).

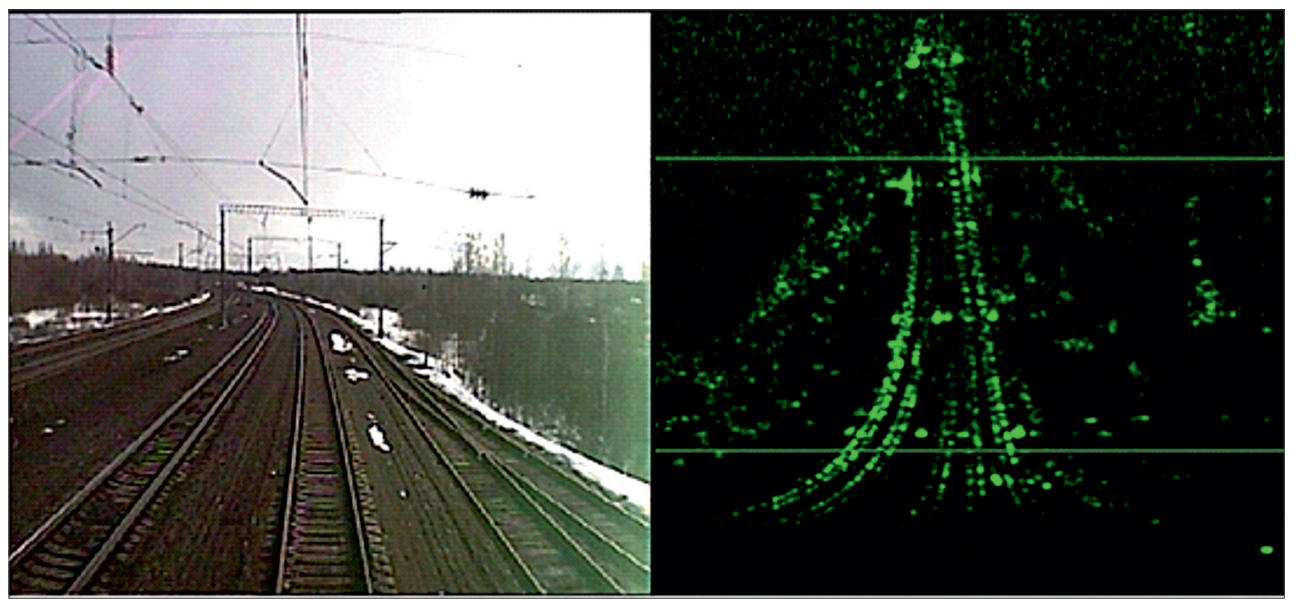

Fig. 9. OI and RI images of railway tracks, at speed.

- enable detection and observation of obstacles such as locomotives, wagons and other objects.

The tests carried out with the use of the experimental RVS model installed on an electric train, both in stationary mode and moving modes, confirmed ability to ensure remote detection of obstacles on the railway tracks.

\section{Building Interferometric SAR based on RVS}

The experience obtained while working with highly detailed radar images, with the view to ensure higher level of safety in transport, enabled us to consider the next step in the development of the radio vision technology. Further development of RVS should focus on small-size radars with antenna aperture synthesis (SAR) capabilities, which will soon find wide practical use to ensure the safety of ships in icing conditions prevailing in northern regions of the planet, due to their low cost and low expenses associated with the operation of their carriers - small-sized unmanned aerial vehicles (UAVs) [16].

Expanding the range of applications of such SAR systems may offer an additional advantage consisting in measuring, in one pass, the height of the underlying surface, with the use of interferometry methods [17]. The mostobvious areas for application of interferometric small-size SAR are:

- all-weather operational monitoring of the ice situation, to identify dilution and icebergs that threaten navigation, to boost both the safety of naval navigation and to ensure the safety of oil platforms in northern oceans,

- round-the-clock, all-weather operational monitoring of flood zones, ice congestion on rivers, avalanche zones, landslides and mudflows,

- operational monitoring of oil pipelines to identify fistulas (leaks of oil products) and critical deformations caused by subsidence of the ground. 
Such an instrument will be especially effective when obtaining periodical, comparative images of the same section of terrain, in small time intervals, to assess the dynamic character of any emergency situation. Here, small-size interferometric SAR will offer some unquestionable advantages, such as its independence from illumination, meteorological conditions, and the presence of smoke and dust in the atmosphere. The low per-hour cost of the carrier - a small $\mathrm{UAV}$, is important as well.

To create such SAR, RVS equipment must be supplemented by a second, identical receiving path and a second antenna. The receiving path antenna must be vertically spaced in relation to the base, which ensures unambiguous measurements of altitude at the selected range. The SAR design should generate radar images of the underlying surface with a range resolution and azimuth of the order of $1 \mathrm{~m}$ within a $3 \mathrm{~km}$ survey strip, from an altitude of about $1 \mathrm{~km}$. In this case, the angles of incidence of the electromagnetic waves (EMW) on the surface (in the approximation of a plane surface) will equal from 15.5 to $60^{\circ}$. With the antenna's elevation plane beam width of about $15^{\circ}$, the capture bandwidth will range from 430 to $1900 \mathrm{~m}$. The width of the signal spectrum necessary to provide the required range resolution (at the near edge of the capture band) is $300 \mathrm{MHz}$. The estimation of SAR's power while mapping the underlying surface, performed based on the radiolocation formula [17], shows that at the required signal-to-noise ratio $q=20 \mathrm{~dB}$, at the maximum operating range, at the gain of the antenna $G=33.4 \mathrm{~dB}$, at the specific radar cross section (RCS) of the underlying surface $\sigma=-20 \mathrm{~dB}$, the average continuous radiation power of SAR with the selected modulation will be $P=5 \mathrm{~W}$.

The requirements for the SAR antenna system are contradictory. On the one hand, it is necessary to provide sufficient amplification of the useful signal (which requires an increase in the antenna size) and sufficient suppression of the side-lobe maxima of the synthesized aperture. On the other hand, if possible, the real antenna beam must be wide enough to minimize losses from the random bank angle and fluctuations of the course angle during the movement of the air carrier in air streams at low altitude.

Let us define the minimum size of antenna diagram in azimuthal plane $d_{a \min }$ :

$$
d_{a \min }=4 V T_{m}=0.12 \mathrm{~m}
$$

where $V$ - carrier speed $(30 \mathrm{~m} / \mathrm{s})$, and $T_{m}$ - probing signals period (1000 samples per second).

To increase SAR energy, we will choose the azimuth aperture of $0.5 \mathrm{~m}$. The ray beam azimuth width will be about $0.9^{\circ}$. Such a wide azimuth beam allows the use of non-coherent accumulation of forth radar image frames to reduce the level of speckle noise. In this case, the requirement for the accuracy of maintaining the heading angle will be less than $\pm 0.5^{\circ}$. To ensure the required gripping strip, the antenna beam width in the elevation plane should be less than $15.2^{\circ}$, which is provided in the $\mathrm{Ka}$ band by the size of the antenna aperture of approximately $3 \mathrm{~cm}$.
The use of a homodyne receiver (substantially reducing the bandwidth of received signals) and frequency expansion of the received signals relying on the RVS technology, as described in [18], can bypass the problem of creating a broadband amplifier with a wide dynamic range. This result allows for a significant narrowing of the dynamic range of signals at the output of the last cascades of the amplifier.

Let us estimate the band of the beat signal $F_{\sigma}$ to be amplified, digitized, and processed:

$$
F_{\sigma}=\frac{2 \Delta F F_{\mathrm{M}} R}{c}
$$

where $F$ - the HF signal band providing the necessary delay resolution $(300 \mathrm{MHz}), F_{\mathrm{M}}$ - signal modulation frequency ( $1 \mathrm{kHz}), R$ - maximum range to the objects being observed (3740 m), $c$ - speed of light.

In consideration of the above, the necessary bandwidth $F_{\sigma}$ for amplification and processing of the received signals will be $3.7 \mathrm{MHz}$, which enables the use of cheap, light and low-energy demand equipment for amplification, digitization and processing of radar signals.

Let us consider the altitude estimation algorithm, assuming that there are two coupled matrices of the complex amplitudes of the signals received at the antennas $A_{1}(i, j)$ and $A_{2}(i, j)$, in the on-board computer. Indices $i$ and $j$ correspond to discrete range and azimuth readings, respectively. The procedure for multiplying the conjugate complex amplitudes must be realized in the computer. As a result, we obtain the following matrix:

$$
A_{1}(i, j) \cdot A^{*}(i, j)=|a(i, j)|^{2} \cdot \mathrm{e}^{j\left[\varphi_{1}(i, j)-\varphi_{2}(i, j)\right]},
$$

where $|a(i, j)|^{2}$ - power multiplier proportional to the intensity of the signals received on two antennas, or the radio brightness field, and $\Delta \varphi(i, j)=\varphi_{1}(i, j)-\varphi_{2}(i, j)-$ field of phase distribution or phase difference.

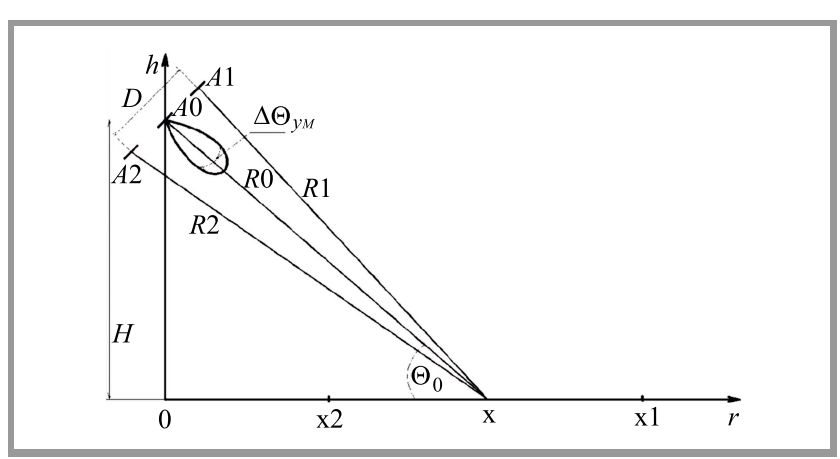

Fig. 10. Geometric relationships in the interferometer.

Consider the dependence of the phase difference of the signals, for a fixed value of the azimuthal coordinate $j=$ constant, and a variable of the inclined range $R=i \cdot \Delta R$. The analysis of this dependence is carried out for different slope angles of the base of the separation of the receiving 
antennas $-\Theta_{0}$, relative to the direction of the local horizontal. The geometrical relationships used are explained in Fig. 10.

It is easy to show that the phase difference $\Delta \varphi(i, j=$ const $)$ will be determined by the expression:

$$
\Delta \varphi(r, j=\mathrm{const})
$$

$$
=k D \cdot\left[\frac{H}{R} \cdot \cos \Theta_{0}-\sqrt{1-\left(\frac{H}{R}\right)^{2}} \cdot \sin \Theta_{0}\right] \text {, }
$$

where: $k=\frac{2 \pi}{\lambda}$ - wave factor, $D$ - receiving antennas spacing base, $H^{-}$- height of the antenna system phase center location over the reflecting surface. The graphs of the normalized phase difference, for various angles - the inclination of the base $-\Theta_{0}$, are shown in Fig. 11. These graphs are obtained in the absence of unevenness of the relief (flat horizontal reflecting surface) and the constant altitude of the flight of the air carrier $(H=1000 \mathrm{~m})$.

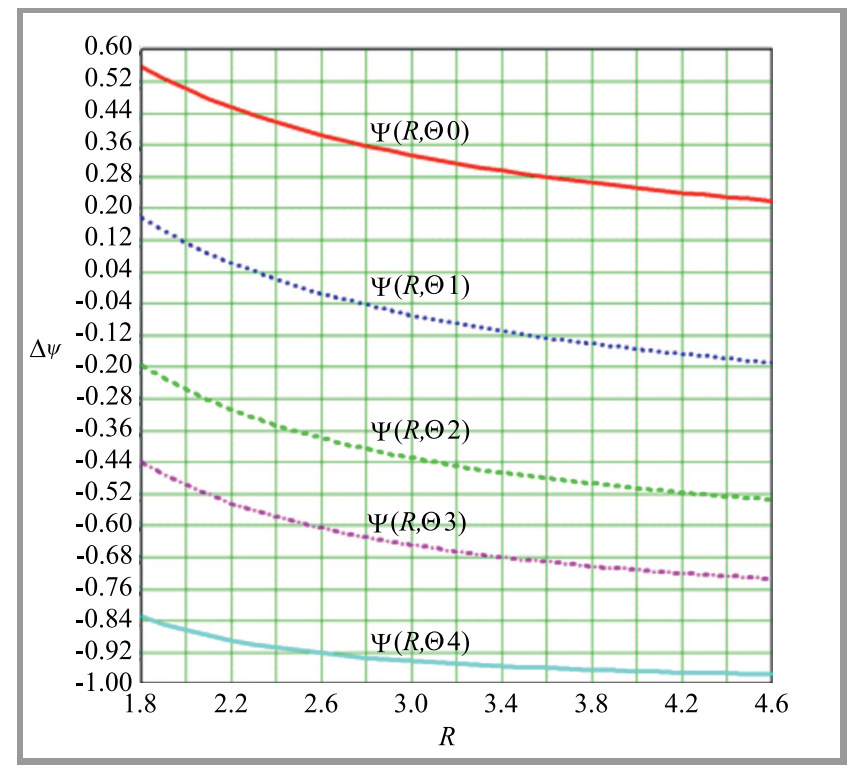

Fig. 11. Normalized dependence of the phase difference (in radians) at a constant altitude $H$ and a variable inclined distance $R$ in $\mathrm{km}$ (for setting angles $\Theta_{0}=0,23.5,45,60$ and $90^{\circ}$ ).

To identify the relationship between the phase difference and the height of the unevenness $h$ (discriminating characteristic), we differentiate the phase difference function with respect to the variable $H$ and write the product of the derivative with variable $h$, which will give us the required characteristic:

$$
\Delta \psi(h)=k D \cdot \frac{H}{R}\left[\cos \Theta_{0}+\frac{\frac{H}{R}}{\sqrt{1-\left(\frac{H}{R}\right)^{2}}} \cdot \sin \Theta_{0}\right] \cdot \frac{h(R)}{H} .
$$

Analysis of the expression shows that the maximum slope of the discrimination characteristic is realized at $\Theta_{0}=0$, increasing the slope angle to $\Theta_{0}=90^{\circ}$ leads to a decrease in the slope and, accordingly, to a decrease in the sensitivity of the interferometer. The slope decreases inversely with the range at which the estimated elevation of the relief is above the average level. An example of a discriminatory characteristic for the setting angles $\Theta_{0}=0,23.5,45$, 60 and $90^{\circ}, \frac{D}{\lambda}=20$ for medium range $R=3600 \mathrm{~m}$, and $H=1000 \mathrm{~m}$, is shown in Fig. 12.

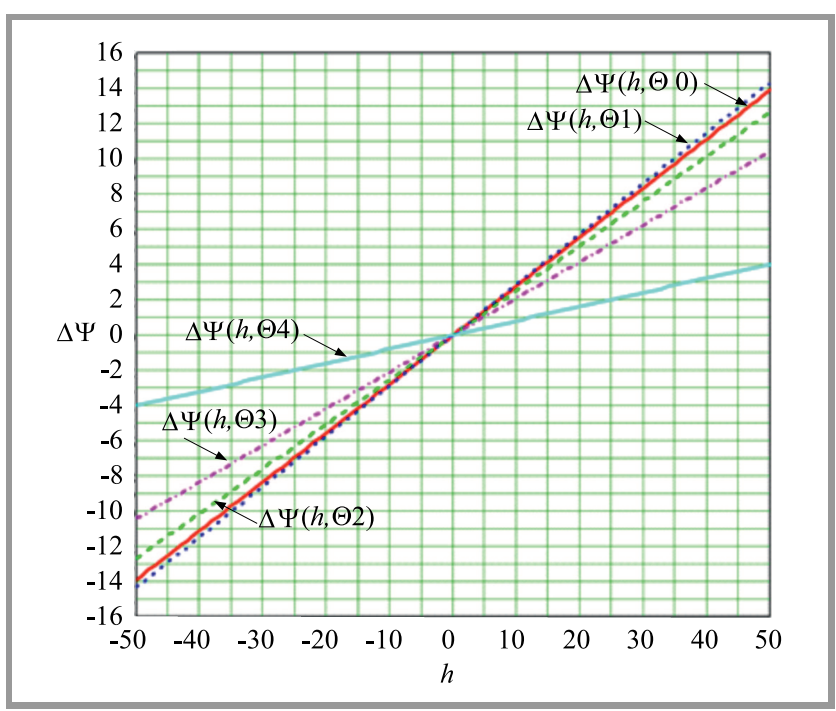

Fig. 12. Discriminatory characteristic (the dependence of the phase difference in radians from the height of the surface in $\mathrm{m}$ ) for the angles $0,23.5,45,60$ and $90^{\circ}$.

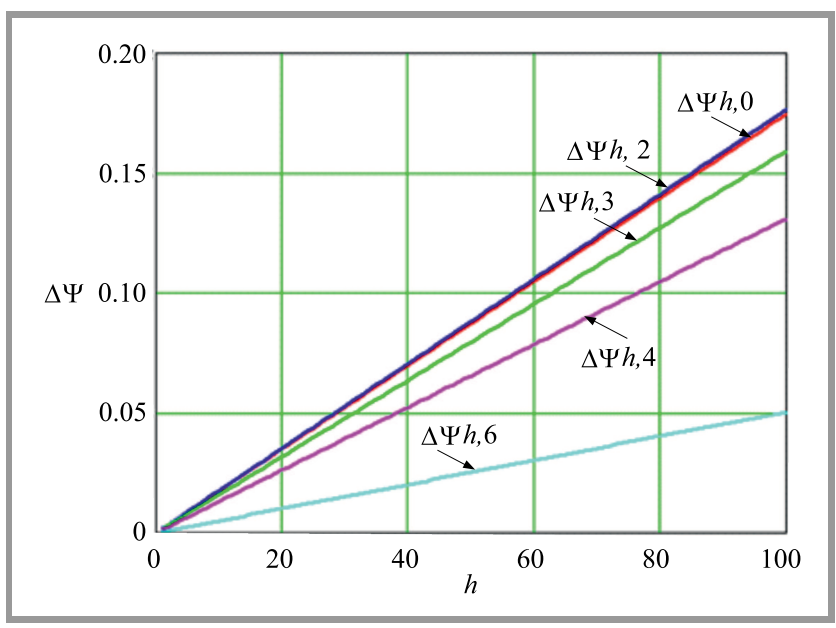

Fig. 13. Normalized (to $\frac{D}{\lambda}$ ) discriminatory characteristics.

Figure 13 shows that normalized discrimination characteristics are constructed as functions of the inclined distances for a fixed value $\frac{h}{H}=0.1$, where the inclination angle of the interferometer base $\Theta_{0}$ is used as the variable parameter.

The results of discriminatory characteristics analysis show that with vertical spacing between the receiving antennas, the maximum sensitivity of the interferometer changes along with the height of irregularities. However, at large bases $\frac{D}{\lambda}=20 \div 30$, the ambiguity of estimates of the current phases is encountered. The use of the horizontal lo- 
cation $\Theta_{0}=90^{\circ}$, leads to a significant decrease in the sensitivity of the interferometer, which can be increased by applying significant separation bases $\frac{D}{\lambda}>30$.

Changing the angle of inclination by $\Theta_{0}=0 \div 60^{\circ}$ does not reduce the sensitivity of the interferometer. Hence, we can consider the angle of $\Theta_{0}=60^{\circ}$ to be the operating inclination angle of the antenna base.

\section{Conclusion}

The article deals with the application of a radio vision system (RVS), which is the optimal all-weather sensor improving the safety of road traffic, aviation, as well as rail and water transport. An example presenting the use of RVS to improve the safety of a modern small aircraft is given. The aircraft-grade RVS will prevent mid-air collisions and will enable to safely perform low-altitude flights, takeoffs and landings in difficult weather conditions, with limited visibility.

Directions in which long-term development of the RVS technology used in railway transport should be heading are formulated. The form and the main technical characteristics proposed for the use of RVS in water transport are presented - enabling all-weather operational monitoring of ice conditions, identifying dilution and icebergs that threaten navigation, both to increase the safety of maritime navigation and that of oil rigs in the world's northern oceans.

\section{Acknowledgements}

This article is based on the results of a research project titled "Creation of scientific and technical reserve solutions in the form of a unified, miniature, low-weight on-board radar for small-size unmanned aerial vehicles to monitor ice conditions during the construction and operation of oil and gas rigs". Agreement no. 14.577.21.0226. Unique identifier RFMEFI57716X0226.

\section{References}

[1] Traffic Safety Facts Research Note NHTSA HS 812101, "2013 Motor Crashes: Overview", Dec. 2014 [Online]. Available: https://crashstats.nhtsa.dot.gov/Api/Public/ViewPublication/812101

[2] Y. Asano, S. Ohshima, M. Ogawa, and K. Nishikawa, "Proposal of millimeter-wave holographic radar with antenna switching", in Proc. IEEE MTT-S Int. Microw. Symp. Digest, Phoenix, AZ, USA, 2001 (doi: 10.1109/MWSYM.2001.967086).

[3] M. Chiaberge (Ed.), New Trends and Developments in Automotive System Engineering. Intech, 2011 (doi: 10.5772/552, ISBN: 9789533075174)

[4] Maria S. Greco, "Automotive radar", in IEEE Radar Conf. RADAR 2012, Atlanta, USA, 2012 [Online]. Available: http://www.iet.unipi.it/m.greco/esami_lab/Radar/ automotive_radar.pdf

[5] K. Shirakawa, S. Kobashi, Y. Kurono, M. Shono, and O. Osamuisaj, "3D-scan millimeter-wave radar for automotive application", Fujitsu TEN Tech. J., no. 38, pp. 3-7, 2013.

[6] L. Stanislas and T. Peynot, "Characterization of the Delphi Electronically Scanning Radar for robotics applications", in Proc. of Australasian Conf. on Robot. and Autom. ACRA 2015, Canberra, Australia, 2015, pp. 434-443.

[7] M. Kishida, K. Ohguchi, and M. Shono, "79 GHz band high-resolutions millimeter-wave radar", Fujitsu Scient. and Tech. J., vol. 51, no. 4, pp. 55-59, 2015.

[8] "Implementing digital processing for automotive radar using SoC FPGAs", White Paper, no. WP-01183-1.2, Altera Corp., 2013.

[9] C. Waldschmitt and H. Meinel, "Future trends and directions in radar concerning the application for autonomous driving", in Proc. of the 11th Eur. Radar Conf. EuRAD 2014, Rome, Italy, 2014, pp. 416-419 (doi: 10.1109/EuRAD.2014.6991296).

[10] A. E. Ananenkov et al., "MM range ARVS as a means of investigating into the objects of natural and and artificial origin", Elektronika i Informatika, no. 4, 2002 [in Russian].

[11] A. E. Ananenkov, A. V. Karpyshev, V. M. Nuzhdin, V. V. Rastorguev, V. B. Shnaider, and G. A. Morozov, "Microwave distance sensor of the helicopter fire fighting system", Russian Aeronautics, vol. 57, no. 4, pp. 406-411, 2014 (doi: 103103/S1068799814040151).

[12] A. E. Ananenkov, A. V. Konovaltsev, V. M. Nuzhdin, V. V. Rastorguev, and P. V. Sokolov, "Optical data-bus and microwave systems for automotive application in vehicles, airplanes and ships", in $O p$ tical and Microwave Technologies for Telecommunication Networks, O. Strobel, Ed. Wiley, 2016 (ISBN: 9781119971900).

[13] Y. V. Likharev, V. M. Nuzhdin, P. V. Sokolov, V. I. Akhrameev, and M. C. Shelagurova, "Determining actual aircraft positions relative to the runway using the airborne radar and approach navigation", Int. J. of Control Theory and Appl., vol. 9, no. 30, pp. 83-93, 2016.

[14] A. Ananenkov, Y. Likharev, V. Rastorguev, and P. Sokolov, "Research of opportunities of short-range radar to prevent flight accidents", in Proc. of 18th Int. Conf. on Transparent Opt. Netw. ICTON 2016, Trento, Italy, 2016 (doi: 10.1109/ICTON.2016.7550247).

[15] "All weather radar IDS-76", DOK Company, 2002-2018 [Online]. Available: http://dokltd.ru/products/a20192

[16] A. E. Ananenkov, A. I. Kanashchenkov, V. M. Nuzhdin, V. V. Rastorguev, and A. M. Smolyar, "Estimation of potential characteristics of onboard radar for ice surface monitoring", in Proc. of 19th Int. Conf. on Transparent Opt. Netw. ICTON 2017, Girona, Spain, 2017 (doi: 10.1109/ICTON.2017.8025027).

[17] M. I. Skolnik (Ed.), Radar Handbook, 3rd Ed. McGraw-Hill, 2008 (ISBN: 9780071485470).

[18] A. E. Ananenkov, A. V. Konovaltsev, V. M. Nuzhdin, V. V. Rastorguev, and P. V. Sokolov, "Homodyne radar", patent no. RU2626405, Russia, 27/07/2017.

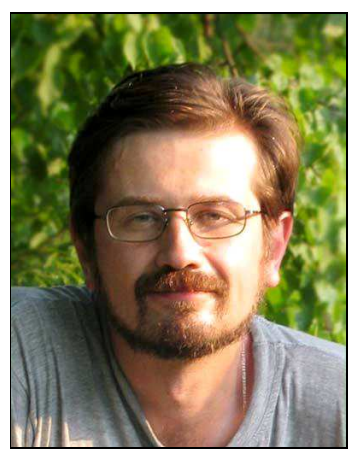

radar systems.
Andrey E. Ananenkov is a senior scientific employee of the Scientific Center for Special Radio-Electronic Systems and Management of the Moscow Aviation Institute - National Research University. $\mathrm{He}$ obtained his Ph.D. from MAI in 1999. He is the author of over 30 scientific publications focusing on signal processing in

E-mail: pan_angej@rambler.ru

Moscow Aviation Institute (National Research University) 4, Volokolamskoye shosse 125993, Moscow, Russia 


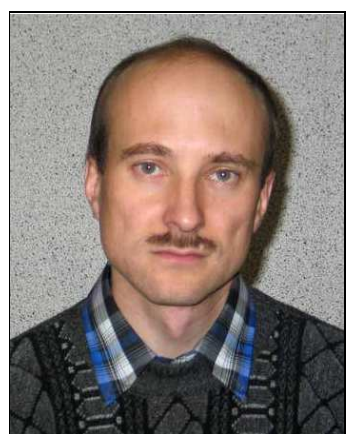

Anton V. Konovaltsev is the leading engineer of the Scientific Center for Special RadioElectronic Systems and Management of the Moscow Aviation Institute - National Research University. He is the author of 27 scientific publications focusing on hardware and software used for processing signals in radio-electronic systems.

E-mail: anton_ko@mail.ru Moscow Aviation Institute (National Research University) 4, Volokolamskoye shosse 125993, Moscow, Russia

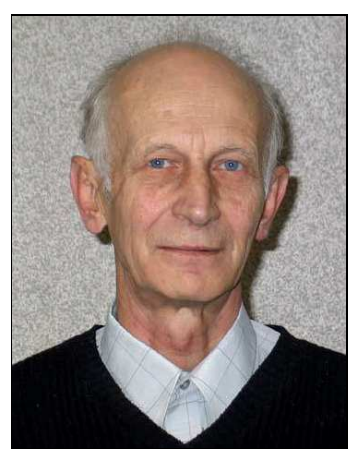

Vladimir M. Nuzhdin, D.Sc., works at the Scientific Center for Special Radio-Electronic Systems and Management of the Moscow Aviation Institute National Research University. He obtained his Ph.D. from MAI in 1983. He is an expert in the area of radar and radionavigation systems and the author of more than 75 scientific

publications.

E-mail: kaf407@mai.ru

Moscow Aviation Institute (National Research University)

4, Volokolamskoye shosse 125993, Moscow, Russia

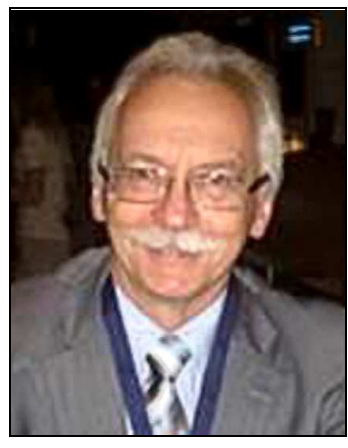

Vladimir V. Rastorguev was the Deputy Director and Professor at the Scientific Center for Special Radio-Electronic Systems and Management of the Moscow Aviation Institute National Research University. $\mathrm{He}$ obtained his Ph.D. from MAI in 1978. He was an expert in the area of optimal signal processing in radar and radionavigation systems. He was also the author of more than 170 scientific publications.

Professor Vladimir V. Rastorguev passed away on December 10, 2018.

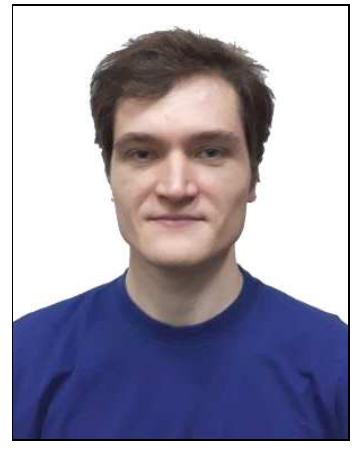

Pavel V. Sokolov is a researcher at the Scientific Center for Special Radio-Electronic Systems and Management of the Moscow Aviation Institute National Research University. $\mathrm{He}$ is an expert in the area of signal processing software used in radio-electronic systems. He is also the author of 25 scientific papers.

E-mail: VerbludSlon@list.ru

Moscow Aviation Institute (National Research University)

4, Volokolamskoye shosse

125993, Moscow, Russia 